\title{
Tool Life Prediction of Ti [C,N] Mixed Alumina Ceramic Cutting Tool Using Gradient Descent Algorithm on Machining Martensitic Stainless Steel
}

\author{
Joseph Daniel $\mathrm{S}^{1}$ and Senthil Kumar $\mathrm{A}^{2}$ \\ ${ }^{1}$ Department of Mechanical Engineering, Mepco Schlenk Engineering College, Sivakasi, India. \\ ${ }^{2}$ Department of Mechanical Engineering, Sethu Institute of Technology, Kariapatti, India. \\ *Corresponding author:asenthil123@gmail.com
}

\begin{abstract}
In automated manufacturing systems, most of the manufacturing processes, including machining, are automated. Automatic tool change is one of the important parameters for reducing manufacturing lead time. Machining studies on Martensitic Stainless Steel was conducted using Ti[C,N] mixed alumina ceramic cutting tool. Tool life was evaluated using flank wear criterion. The tool life obtained from experimental machining process was taken as training dataset and test dataset for machine learning. Tool life model was developed using Gradient Descent Algorithm. The accuracy of the machine learning model was tested using the test data, and $99.83 \%$ accuracy was obtained.

Copyright (C) 2020. Journal of Mechanical Engineering Science and Technology.

All rights reserved.
\end{abstract}

Keywords: Gradient descent algorithm, machine learning, machining, prediction, tool life model.

\section{Introduction}

Alumina based ceramic cutting tools have unique chemical, and mechanical properties and these tools can offer increased metal removal rates, extended tool life and the ability to machine hard workpiece materials like hardened steel and stainless steel. The ceramic cutting tools can reduce the cost of machining and increase productivity because of their high material removal rates [1]. Alumina based ceramic cutting tools are capable of machining various types of hard materials due to the improved cutting tool properties such as fracture toughness, thermal shock resistance, hardness and wear resistance. The advantages of using ceramic cutting tools are that, the hard materials like hardened steels, stainless steels and hard powder metal materials with complex shapes can be machined in their hardened conditions. The grinding quality surface finish can be obtained by turning the hard work materials using ceramic cutting tools.

The properties of Aluminium oxide are enhanced by the addition of titanium carbide (TiC) in the alumina matrix, which increases the transverse rupture strength and thermal shock resistance of the composite tool. The titanium nitride (TiN) is also used as a secondary ceramic phase because of its superior thermal conductivity. By adding these non-oxide particles like $\mathrm{TiC}$ and $\mathrm{TiN}$ in the alumina matrix, the thermal conductivity, the thermal shock resistance and the hardness are increased. These composite ceramic cutting tools retain their hardness even at elevated temperature. In the Ti[C, N] mixed alumina composite ceramic cutting tool, the TiC, TiN grains pin the crack initiated in the matrix [2]. The toughening mechanism for this type of mixed ceramic cutting tools is known as precipitate or dispersion strengthening. Mixed alumina based ceramic tools are fabricated by hot pressing, which 
involve mixing of fine grained alumina with $20-30 \%$ volume of $\mathrm{TiC}$ and $\mathrm{TiN}$ powders. These ceramic cutting tools are generally used for machining of hardened steels because of their increased hardness.

Martensitic Stainless steels are iron alloys with a minimum of $11.5 \%$ chromium. In addition to iron, carbon, and chromium, stainless steel may also contain other elements, such as nickel, niobium, molybdenum, and titanium. The chromium content in stainless steel enhances the corrosion resistance. Martensitic stainless steels are magnetic, contains higher carbon content than the ferritic types. They are hardenable by quenching and tempering like plain carbon steels and find their main application in cutlery, surgical tools, aerospace and general engineering. Ronald Klueh and Donald Harries (2001) have reported that advanced ferritic/martensitic stainless steel is used in thermal power plants, nuclear power plants and in other demanding environments for its high temperature properties, and high creep rupture strength [3]. Grade ASTM A276 is the basic martensitic stainless steel, and like most nonstainless steels it can be hardened by a "quench-and-temper" heat treatment. In the annealed or highly tempered conditions grade ASTM A276 machined without much difficulty, but if hardened to above $30 \mathrm{HRC}$ machining becomes very difficult. Stainless steel grade ASTM A276 is used for parts requiring a combination of good strength, toughness and reasonable corrosion resistance and typical applications include bolts, nuts, screws, bushings, pump and valve parts, shafts, steam turbine parts, gas turbine parts, petrochemical equipment, mine equipment etc. In this present work, the tool life of $\mathrm{Ti}[\mathrm{C}, \mathrm{N}]$ mixed alumina based ceramic cutting tools is evaluated on machining hardened martensitic stainless steel - grade ASTM A276.

Tool life and tool wear prediction have been attempted by many researchers using various tools and machine learning algorithms. Artificial Neural Network has been widely used to predict tool wear and tool life. Mikołajczyka et al. used Artificial Neural Network (ANN) and trained them using the data subset obtained from actual machining and a predicted data subset obtained from image recognition. The trained ANN is used to evaluate the tool life in turning operations of a third test set [4]. Gouarir et al. used sensors to continuously monitor and measure the flank wear and adaptive control (AC) along with Convolutional Neural Networks (CNN)was used to predict tool wear [5]. Xuefeng Wu et al. used ANN to monitor the tool wear from the tool wear data obtained through cameras. A Convolutional Automatic Encoder (CAE) is used to train the neural network with data obtained from the camera. Backpropagation and stochastic gradient descent are performed to obtain average recognition precision rate of $96.20 \%$ [6]. Apart from ANN, the researchers used Support Vector Machines, Logistic Regression, Random Forest algorithms to predict tool wear and tool life. Jaydeep Karandikar et al. used Support Vector Machines and Logistic Regression methods to predict the tool wear characteristics of a given tool and to model the tool life [7]. Schwenzer et al. used Support Vector Machine (SVM) and random forest algorithms on datasets obtained from orthogonal cutting in milling. They are used to classify the tool as 'sharp' or 'dull' with the help of force and current signals obtained from sensors [8]. Yang Hui et al. used Support Vector Machine (SVM) algorithm to extract the features from the vibration signals are sensed from a milling tool. The stacked generalization (SG) ensemble model based on SVM, decision tree (DT), Naive Bayes (NB) algorithms are used to recognize the tool wear state of the milling tool [9]. Benjamin Neef et al. used SVM and random forest ensemble (RSE) algorithms to analyse the high frequency current samples of a CNC turning machine terminal to estimate of the tool wear. Experimental studies are conducted and the accuracy of the machine learning model is noted. An online continuous tool wear monitoring system is proposed for easy tool wear monitoring [10]. Dazhong Wu 
used Cloud computing, Industrial Internet of Things (IIoT) and machine learning to estimate the tool wear characteristics of a cutting tool. Random forests (RF) algorithm was used alongside 'MapReduce' data processing scheme and the training time is reduced by 14.7 times along with a high prediction accuracy [11]. In addition to machine learning algorithms, signal and image processing were also used to predict tool wear. Giovanna Mart inez et al. used signal imaging to encode the images of the tool at specified time steps and fed to a premade deep learning package for classifying the tool wear as break-in wear, steady wear, severe wear and failure region [12]. Bovic Kilundua et al. measured vibration signals on the tool holder and pseudo-local singular spectrum analysis was done to extract the features that are essential for the quality of the tool and is monitored continuously [13]. Even though the researchers attempted various machine learning algorithms, few has attempted linear algorithms. Most of them used classification for predicting the status of the tool. Linear algorithms are simple, but powerful tools for modelling. Gradient Descent Algorithm (GDA) is one of the linear algorithms widely used in various types of modelling. An attempt has been made to predict tool life using GDA by training them using the data obtained from machining hardened and tempered martensitic stainless steel - grade ASTM A276 by Ti $[\mathrm{C}, \mathrm{N}]$ mixed alumina based ceramic cutting tool.

\section{Materials and Methods}

\section{A. Cutting Tool Inserts}

Machining tests were carried out using $\mathrm{Ti}[\mathrm{C}, \mathrm{N}]$ mixed alumina ceramic cutting tool inserts on a precision lathe with variable spindle speeds and feeds. The specifications of the cutting tool inserts are presented in Table1.

Table 1. Details of cutting tool inserts specifications

\begin{tabular}{cccccc}
\hline $\begin{array}{c}\text { Insert } \\
\text { specification } \\
\text { (ISO) }\end{array}$ & Shape & $\begin{array}{c}\text { Rhombic nose } \\
\text { angle }\end{array}$ & $\begin{array}{c}\text { Rhombic inscribed } \\
\text { circle diameter }\end{array}$ & Thickness & $\begin{array}{c}\text { Nose } \\
\text { radius }\end{array}$ \\
\hline $\begin{array}{c}\text { CNGN } \\
120408 \mathrm{~T} 01020\end{array}$ & Rhombic & $80^{\circ}$ & $12.7 \mathrm{~mm}$ & $4.76 \mathrm{~mm}$ & $0.8 \mathrm{~mm}$ \\
\hline
\end{tabular}

\section{B. Work Materials}

The work material used in these machining studies was martensitic stainless (ASTM A276) steel and was hardened and tempered to HRC 42. Machining studies were conducted on them. The composition of the stainless steel (ASTM A276) is given in Table 2.

Table 2. Composition of Stainless steel - ASTM A276 grade by weight percentage

\begin{tabular}{ccccccccc}
\hline Elements & $\mathrm{C}$ & $\mathrm{Si}$ & $\mathrm{Mn}$ & $\mathrm{Cr}$ & $\mathrm{Ni}$ & $\mathrm{P}$ & $\mathrm{S}$ & $\mathrm{Fe}$ \\
\hline Weight Percentage & $0.09-0.15$ & 1.0 & 1.0 & $11.5-13.5$ & 1.0 & 0.04 & 0.03 & Balance \\
\hline
\end{tabular}

\section{Experimental Conditions}

Machining studies were conducted on hardened martensitic stainless steel-grade ASTM A276 using Ti $[\mathrm{C}, \mathrm{N}]$ mixed alumina based ceramic cutting tool at different cutting speeds and at constant feed rate and depth of cut. Experimental conditions are shown in Table 3. 
Table 3. Experimental conditions

\begin{tabular}{lll}
\hline Cutting speed (V) & $\mathrm{m} / \mathrm{min}$. & $\begin{array}{l}100,120,140,160,180, \\
220,240,260,280,300\end{array}$ \\
\hline Feed rate (f) & $\mathrm{mm} / \mathrm{rev}$. & 0.12 \\
\hline Depth of cut (d) & $\mathrm{mm}$. & 0.5 \\
\hline Environment & & Dry \\
\hline
\end{tabular}

\section{Observations on Tool Wear and Tool Life}

The main objective of the present study is to evaluate the tool life of $\mathrm{Ti}[\mathrm{C}, \mathrm{N}]$ mixed ceramic cutting tools on machining ASTM A276 steel (HRC 42) by measuring tool wear. The average flank wear measurement was observed from five machining tests. The wear measurements were taken using a toolmakers microscope (Metzer-model METZ 1395) with $30 \mathrm{X}$ magnification factor. The machining time was accurately measured with a stopwatch. Flank wear is one of the main types of wear generally occur while machining hard materials. The machining was stopped periodically to measure flank wear of the cutting tool. The tool life of the cutting tool is considered as per 'ISO Standard 3685 for tool life testing' and it is the machining time of the cutting tool when the average flank wear reaches $0.4 \mathrm{~mm}$. The tool life of the $\mathrm{Ti}[\mathrm{C}, \mathrm{N}]$ mixed ceramic cutting tool was found out by observing the flank wear of the cutting tool at various cutting speeds.

\section{E. Tool Life Model}

Tool life model has been developed using GDA. It minimizes an objective function and iterates several time to minimize error. The algorithm updates the model after each iteration and finally converges into local minima. The learning rate is used to specify the number of steps required to reach the local minima. The machining data obtained from turning operation was used to train the model. The trained model was used to predict tool life. The tool life of Ti $[\mathrm{C}, \mathrm{N}]$ mixed ceramic cutting tool on machining ASTM A276 steel were found out. Using the tool life data, tool life models were developed using GDA. For comparison, the regression model for tool life has also been developed using least square method (LSM).

\section{Results and Discussions}

Machine Learning algorithms learn from the data and predict the output without human intervention. There are several types of machine learning algorithms and linear algorithms are used where the input parameters and output variables exhibit a linear relationship. The aim of the linear algorithm is to find the best-fit model by training the algorithm with given input parameters. The linear algorithms try to minimize the error of prediction finds the appropriate model which has minimum errors. Gradient descent is one of the linear algorithms which uses minimization technique. The GDA trains the machine learning model and iterates a number of times until it converges into a local minima. Tool life prediction plays an important role in the machines that are connected to Automated Manufacturing System (AMS). The change of cutting tool insert should happen at predicted times. So, tool life prediction is an important process in automated systems and the machine learning algorithms play vital role in automation. Using the experimental machining data, the tool life model was developed using GDA. In addition to the machine learning model, tool life model using conventional LSM was also developed for comparison. Machining studies carried out and experimental data of the life and cutting speed plotted in Figure1. 


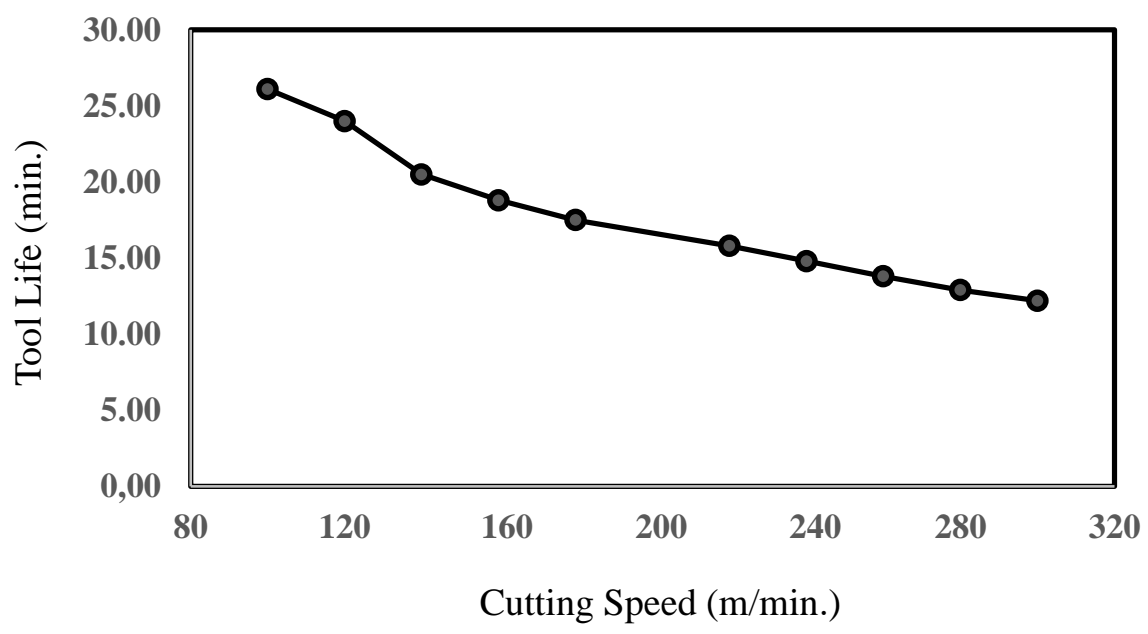

Fig. 1. Cutting Speed vs. Tool life of Ti $[\mathrm{C}, \mathrm{N}]$ mixed ceramic cutting insert

\section{A. Tool Life Model Using Gradient Descent Algorithm}

Tool life model using GDA was developed. GDA works well, if the dependent variables and independent variables have a linear relationship. The machine learning model using GDA was developed using Taylors's equation $\mathrm{VT}^{\mathrm{n}}=$ constant. This equation can be slightly modified to have linear relationship.

$\mathrm{VT}^{\mathrm{n}}=\mathrm{C}$

By taking logarithm,

$\log \mathrm{V}+\mathrm{n} \log \mathrm{T}=\log \mathrm{C}$

By rearranging,

$\log \mathrm{T}=(1 / \mathrm{n}) \log \mathrm{C}-(1 / \mathrm{n}) \log \mathrm{V}$

where $\mathrm{V}$ - cutting speed in $\mathrm{m} / \mathrm{min}$; $\mathrm{T}$ - tool life in minutes; $\mathrm{C} \& \mathrm{n}$ - constants

The above equation can be rewritten in the form of $y=a+b x$, which represents the logarithmic linear relationship between cutting speed and tool life. The tool life found out from the experimental machining studies were used to develop the tool life models. Using GDA, the tool life model was developed and the constants of the models were found out.

The GDA iterates and finds out best possible model with minimum error. The algorithm was trained to predict using the given input independent variable ' $x$ ' and the output dependent variable ' $y$ ' tabulated in Table 4. From the dataset given in the table, the tool life model using GDA was developed. Even though dataset contains less variables, it is the sample tool life model and similar larger number of industrial datasets can be used to develop tool life model with same accuracy. 
Table 4. Machining Dataset of input variable ' $x$ ' and the output variable ' $y$ '

\begin{tabular}{lll}
\hline S.No & Input variable 'x' & Output variable ' $y$ ' \\
\hline 1. & 2 & 1.41664051 \\
2. & 2.07918125 & 1.38021124 \\
3. & 2.14612804 & 1.31175386 \\
4. & 2.20411998 & 1.27415785 \\
5. & 2.25527251 & 1.24303805 \\
6. & 2.34242268 & 1.19865709 \\
7. & 2.38021124 & 1.17026172 \\
8. & 2.41497335 & 1.13987909 \\
9. & 2.44715803 & 1.11058971 \\
10. & 2.47712125 & 1.08635983 \\
\hline
\end{tabular}

The model was trained using GDA and for every iteration, Root Mean Square Error (RMSE) was found out. The number of iterations was more as smaller dataset needed more training time. The RMSE vs. Number of Iterations is depicted in Figure2. The machine learning algorithm iterations were carried out with a learning rate of 0.3 , until it reached local minima. The iterations were stopped when the next iteration RMSE value was greater than the current iteration value. The local minima was converged at $23610^{\text {th }}$ iteration. The learning rate of the machine learning algorithm was varied to 0.1 and 0.2 , to analyse the effect of learning rate for convergence to local minima. The convergence point of the local minima was observed and it was plotted in Figure3. For the same dataset tool life model was developed using LSM.

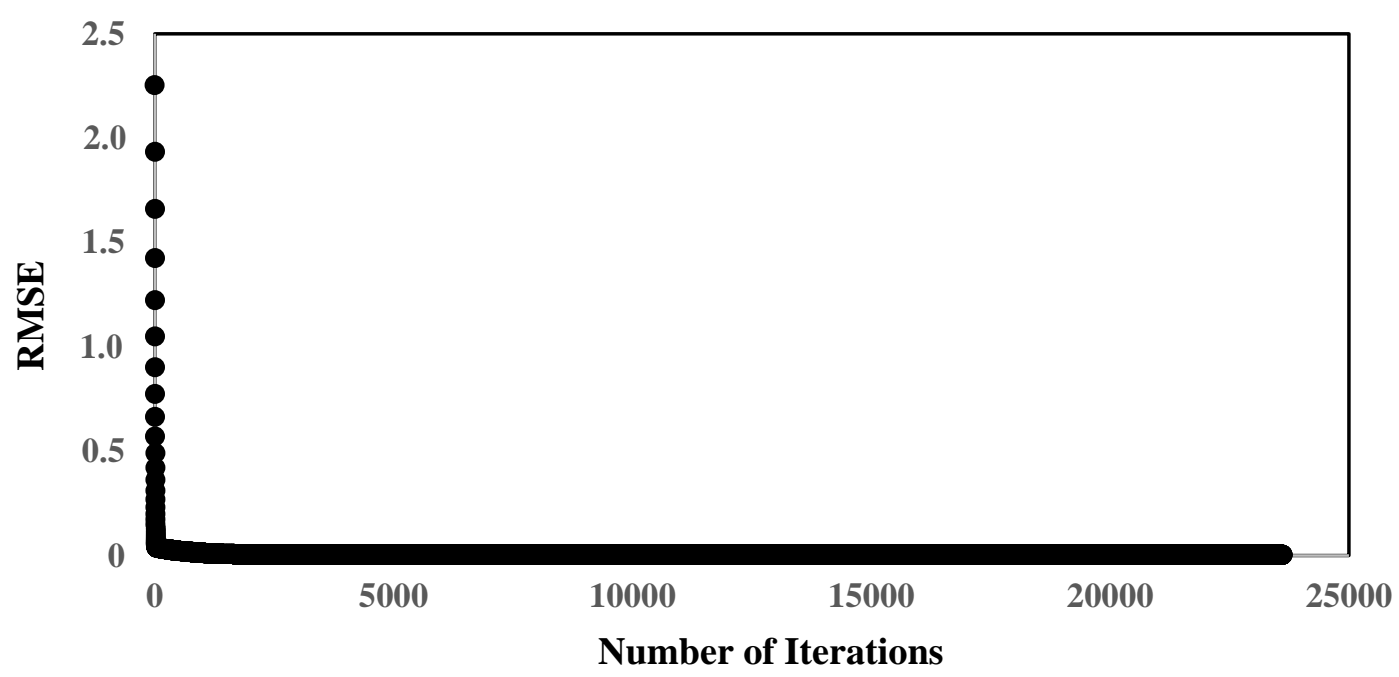

Fig. 2. RMSE vs. Number of Iterations

The tool life models developed using GDA and LSM are given below.

Gradient Descent Algorithm (GDA): Y $=2.788458-0.683752$ X 


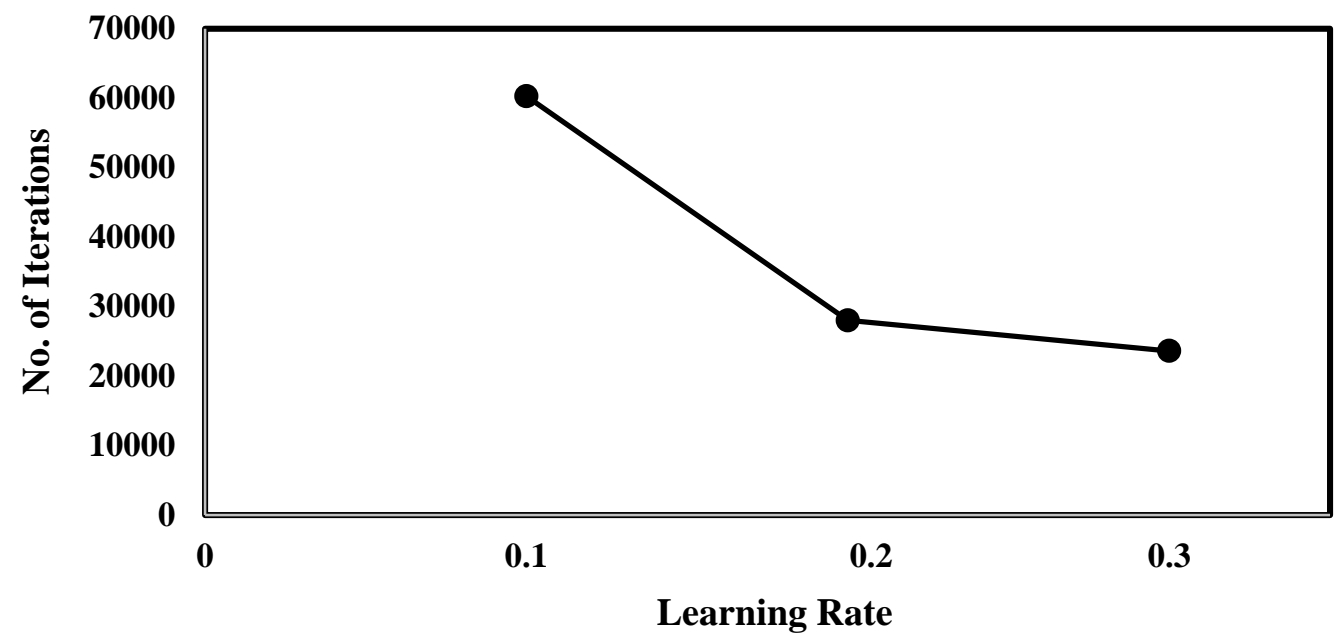

Fig. 3. Number of iterations required for convergence vs. Learning rate

\section{B. Comparison of the Tool Life Models}

The tool life model based on GDA and the tool life model based on LSM were compared for the RMSE. It is used to measure the difference between the predicted values and the observed or actual values. RMSE is a measure of the spread out of the errors from the regression line. The RMSE of the tool life model using GDA and that of the tool life model using LSM is compared in Figure4. From this figure, it can be observed that the tool life model using GDA has lower RMSE than the tool life model using LSM. It is also can be observed that the RMSE error is very minimum for the GDA tool life model. The validity and significance of the model was found out using coefficient of determination. The coefficient of determination is also known as R-squared $\left(\mathrm{R}^{2}\right)$, assesses the linear relationship is between two variables. Similarly, the Adjusted R Squared ( $R^{2}$ Adj.) determines the extent of the variance of the dependent variable by all independent variables. The $\mathrm{R}^{2}$ value and $\mathrm{R}^{2}$ Adj. value of Tool life model using GDA are: $\mathrm{R}^{2}=0.994084$ and $\mathrm{R}^{2} \mathrm{Adj} .=0.99334$. It can be observed that the machine learning model has significance level of $99 \%$.

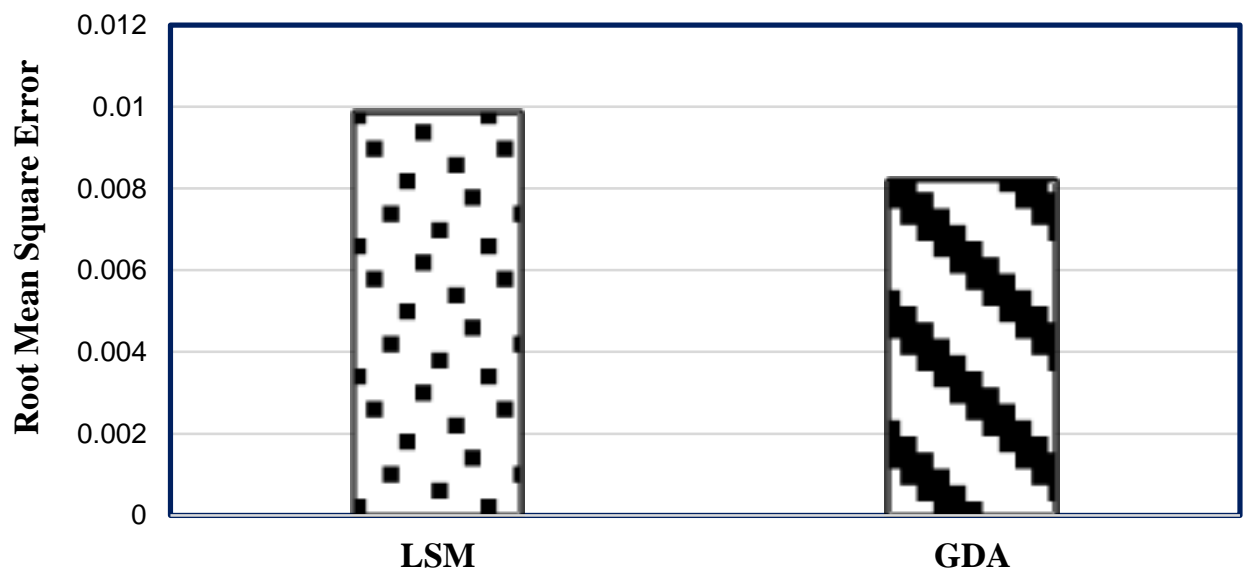

Fig. 4. Comparison of RMSE of tool life models based using GDA and LSM 


\section{Prediction of Tool Life}

In order to validate the machine learning tool life model, machining studies were carried out and tool life were evaluated for various cutting speeds. Using the machine learning tool life model, tool life were predicted for the given cutting speeds. The predicted values for the test data set and the actual values are compared and observed that the percentage of error is very minimum and the error is not more than $0.3 \%$ in the given test.

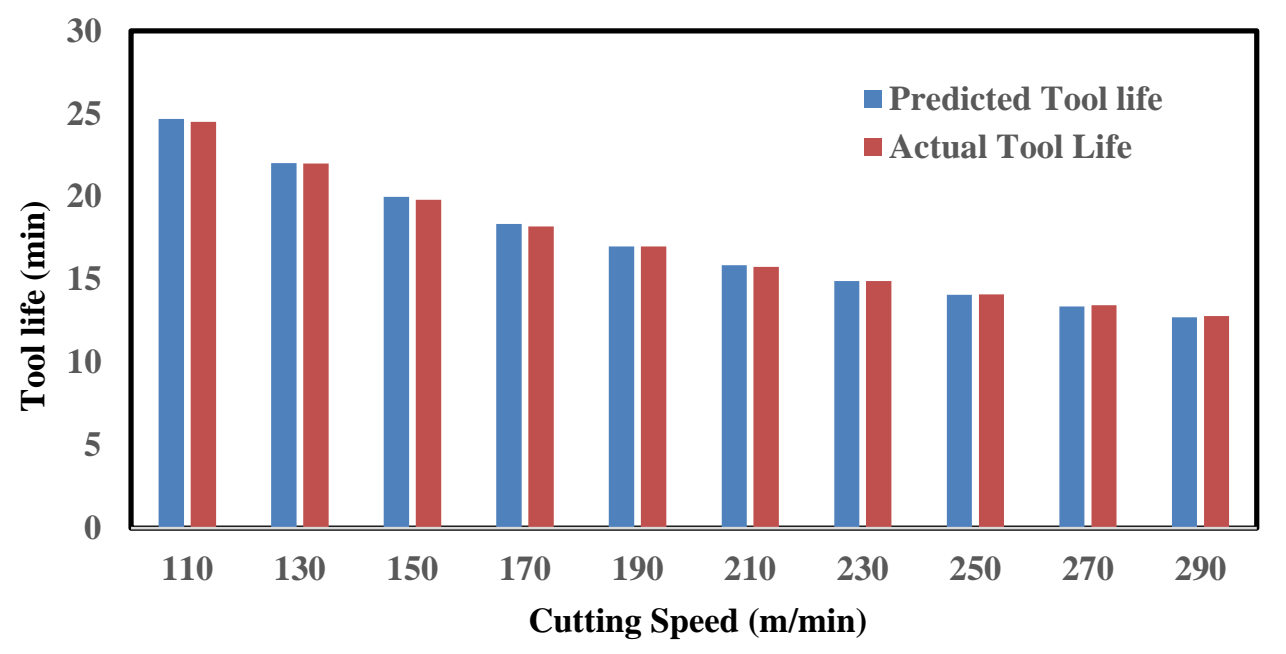

Fig. 5. Comparison of Predicted Tool Life with Actual Tool Life

The average percentage of error is $0.17 \%$ and the accuracy of the model is $99.83 \%$. Even though the model is very simple, it is very effective for predicting tool life. The output data is converted to tool life and the predicted tool life and the actual tool life is presented in Figure5. From this figure, it can be inferred that the predicted tool life values are very close to the actual tool life values. Hence, the GDA can be successfully implemented for tool life prediction.

\section{Conclusions}

Machining studies were conducted using Ti $[\mathrm{C}, \mathrm{N}]$ mixed alumina based ceramic cutting tool on ASTM A276 martensitic stainless steel. The training dataset and test data were obtained by evaluating the tool life experimentally. Tool life model was developed using Gradient Descent Algorithm. For comparison, tool life model based on Least square method was also developed. Different learning rates were attempted to improve the performance of the model. Root Mean Square Error was evaluated with various learning rates and the convergence with minimum number of iterations occurred at a learning rate of 0.3 . The tool life model was validated using $\mathrm{R}$ square and adjusted $\mathrm{R}$ square and it was found that the model had a significance level of $99 \%$. Tool life prediction were carried out using the test data and the model had an accuracy of $99.83 \%$. The predicted tool life values are very close to the actual tool life values. The Gradient Descent Algorithm was successfully implemented for tool life prediction.

\section{References}

[1] Rahul, R., Alok Kumar, D, "A review on cutting of industrial ceramic materials", Precision Engineering, vol. 59, pp. 90-109, 2019. 
[2] Fei, Y.H., Huang, C.Z., Liu, H.L., Zou, B., "Mechanical properties of Al2O3-TiCTiN ceramic tool materials”, Ceramic International, vol. 40 (7), pp. 10205-10209, 2014.

[3] Ronald L. K., and Donald R. H., High chromium ferritic and martensitic steels for nuclear applications, ASTM International, West Conshohocken, Pennsylvania, USA, pp. 5-27, 2001.

[4] Mikołajczyka, T., Nowickib, K., Bustilloc, A., Yu Pimenovd, D., "Predicting tool life in turning operations using neural networksand image processing", Mechanical Systems and Signal Processing, vol. 104, pp. 503 - 513, 2018.

[5] Gouarir, A., Martínez-Arellano, G., Terrazas, G., Benardosand, P., Ratchev, S., "Inprocess Tool Wear Prediction System Based on Machine Learning Techniques and Force Analysis", Procedia CIRP, vol. 77, pp. 501 - 504, 2018.

[6] Wu, X., Liu, Y., Zhou, X., and Mou, A., “Automatic Identification of Tool Wear Based on Convolutional Neural Network in FaceMilling Process", Sensors, vol. 19, pp. 3817, 2019.

[7] Karandikar, J., "Machine learning classification for tool life modeling using production shop-floor tool wear data", Procedia Manufacturing, vol. 34, pp. $446-$ 454, 2019.

[8] Schwenzer, M., Miura, K., and Bergs, T., "Machine Learning for Tool Wear Classification in Milling Based on Force and Current Sensors", IOP Conference Series Materials Science and Engineering Vol. 520, 2019.

[9] Hui, Y., Mei, X., Jiang, G., Tao, T., Pei, C., and Ma, Z., "Milling Tool Wear State Recognition by Vibration Signal Using a Stacked Generalization Ensemble Model", Shock and Vibration, vol. 2019, pp. 7386523, 2019.

[10] Neef, B., Bartels, J., and Thiede, S., "Tool Wear and Surface Quality "Monitoring Using High Frequency CNC Machine Tool Current Signature", 2018 IEEE 16th International Conference on Industrial Informatics (INDIN), 2018.

[11] Wu,D., Jennings, C., Terpenny, J., Kumara, S., and Gao, R.X., "Cloud-Based Parallel Machine Learning for Tool Wear Prediction”, Journal of Manufacturing Science and Engineering, vol. 140(4), pp. 041005, 2018.

[12] Mart inez-Arellano, G., Terrazas, G., and Ratchev, S., "Tool wear classification using time series imaging and deep learning", The International Journal of Advanced Manufacturing Technology, vol. 104, pp. 3647 - 3662, 2019.

[13] Kilundua, B., Dehombreuxa, P., and Chiementin, X., "Tool wear monitoring by machine learning techniques and singular spectrum analysis", Mechanical Systems and Signal Processing, vol. 25, pp. 400 - 415, 2019. 